\title{
Assessment Of Alternative Precision Positioning SySTEMS
}

\author{
Dr. Y. Morgan \\ University of Regina, Canada
}

\begin{abstract}
The continuous evolution of technology, electronics, and software along with the dramatic decrease in the cost of electronic devices has led to the spread of sensing, surveillance, and control devices. The Internetof-Things (IoT) benefits from the spread of devices (things) by processing device feeds using Machine-toMachine (M2M) technologies. At the heart of the M2M technologies lies the ability of devices (things) to identify their own location on the globe or relative to known landmark. Since location awareness is fundamental to processing sensing and control feeds, it has attracted researchers to identify ways to identify and improve location accuracy. The article looks at Global Positioning Systems (GPS) along with the enhancements and amendments that apply to satellite based solutions. The article also looks at medium to short-range wireless solutions such as cellular, Wi-Fi, Dedicated Short-Range Communications (5.9 $\mathrm{GHz}$ DSRC) and similar solutions.

In this article define the physical limitations of current system, we define situation where every particular system underperforms and elaborate the downfalls pertaining to inherent characteristics of the proposed technology.
\end{abstract}

\section{KEYWORDS}

Wireless Network, Mobile Network, Geographical Positioning Systems, Short-Range Wirelss

\section{INTRODUCTION}

Current GPS systems suffer from the low accuracy and from the absence of GPS signal in tunnels and indoor locations. Consequently, number of solutions emerged by typically relying on shortrange wireless solutions. In order to evaluate and compare those solutions, we identify Precision Positioning Systems Evaluation Matrix (PPS-EM). The PPS-EM is designed to assist comparing possible PPS augmenting solutions. Any alternative PPS must exhibit the following characteristics:

- Accuracy: increased precision reduces space for vehicle maneuvers so that throughput is improved in direct relation to accuracy. Desired positioning accuracy should be $1 \mathrm{~cm}$ or less;

- Accuracy Awareness: PPS should be able to estimate position as well as the confidence in estimated position. Knowing the range of error enables other vehicle systems to abort potentially unsafe maneuvers. Typically, each PPS-based application has its own bounded tolerance for errors; 
- Fault Tolerance and Awareness: PPS should be able to revert to available alternative positioning technologies whenever a fault is detected in the system, otherwise, system failure should be declared and safety applications must rely on other means for recovery;

- Security: PPS systems must withstand possible malicious attacks. One way to do that is by increasing system redundancy. Another approach is to rely only on trusted sources of information. A typical secure system would combine several security mechanisms;

- Reliability: Functionality, availability, and accuracy of the system must remain reliable regardless of the physical environment such as weather or road conditions, surroundings (e.g., tunnels and parking facilities) or road visibility;

- Localization: Proposed systems must be localized not centralized. Failure of centralized systems such as GPS may result in failure in transportation systems of multiple cities. Alternatively, failure of localized systems would have limited impact;

- Economic Feasibility: Any PPS for transit ITS application must be economically feasible;

The PPS-EM requirements are difficult to achieve. Hence, we investigate and compare the different approaches pertaining to PPS. For example, embedding radio frequency identification (RFID) circuits or magnetic guidance markers on every roadway in North America may provide the required positional accuracy, but the economic feasibility is uncertain. Image processing solutions to generate visual awareness of lane position are cost efficient, but have questionable reliability in harsh weather environments. Positioning has been the subject of investigation for the cellular and Wi-Fi industries. While some requirements may differ, there are benefits in examining the wireless industry experiences and extracting the lessons learned and best practices as well.

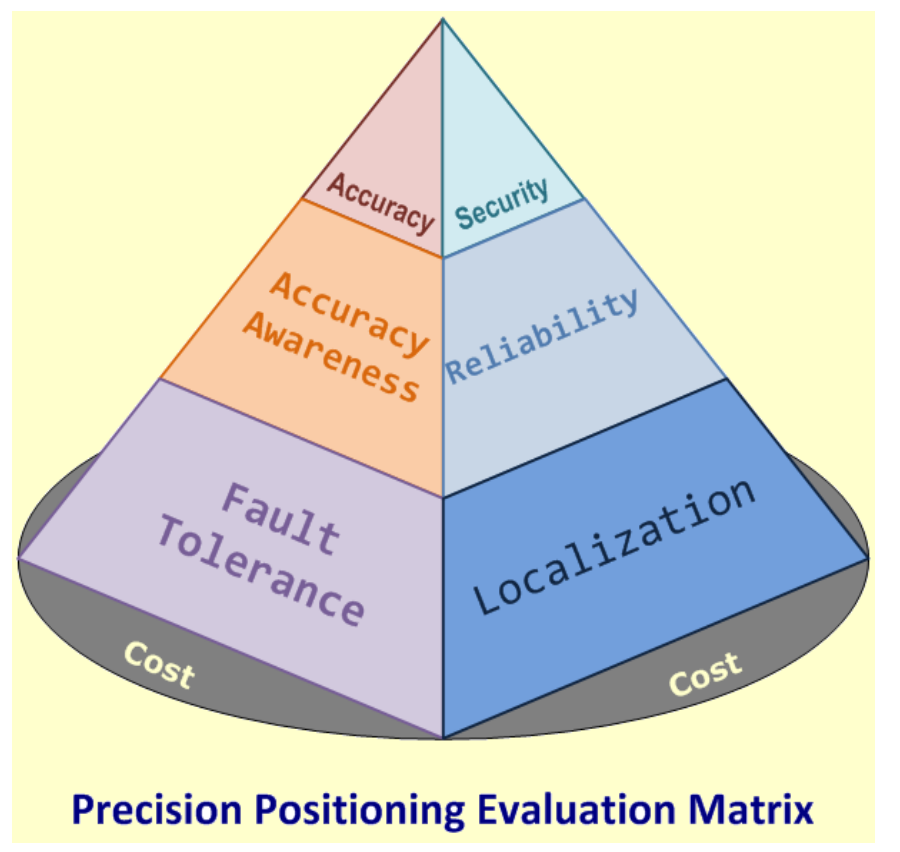




\section{TECHNICAL RERVIEW}

Due to the wide variety of positioning approaches, it is important to categorize those approaches in order to express similarities. The principal of trilateration is discusses in Appendix-I. Trilateration is commonly known as ranging or triangulation, and is highly used for object location in most wireless technologies. Satellite-Based approaches along with alternative enhancements and amendments are discussed in the following section. A view on the limitations of Satellite-Based approaches is added to clarify the rationale behind PPS evolution[3]. Then a discussion of the Medium and Short-Range solutions such as cellular, TV-broadcast, Wi-Fi, and 5.9 GHZ DSRC solution follows. Finally, we present the Embedded Road Markers approaches namely, Magnetic Guidance Systems (MGS), RFID and Ultra Wide-Band (UWB) with a discussion on their economic feasibility[2].

\subsection{Satellite-Based Solutions}

The satellite-based Global Positioning Systems (GPS) have been used for decades for object positioning with an accuracy of about $15 \mathrm{~m}$. Position is typically calculated by a GPS receiver using the position of the visible satellites, the measured delay of the received signals making accuracy primarily dependent on known satellite position and calculated signal delay. The smaller time units lead to better accuracy. However, since the absolute and accurate satellite location is known only at higher aggregates of time, fixes to satellite location are propagated less frequently. Therefore, object positions are influenced by the amount of Time-To-First-Fix (TTFF) as well. For detailed description of Satellite-Based Solutions, please refer to the Technical Approach Chapter.

\subsection{Limitations of Satellite-Based Solutions}

Unfortunately, Satellite-Based systems have been stretched to its limits and needs to be complemented by other positioning technologies to provide reliable metropolitan coverage and improve fix times for a new generation of PPS applications, such as BRT, platooning, and precision docking. Vehicular positioning capabilities are widely regarded as the ultimate goal for PPS with a large number of modern solutions competing to get to market first. Those limitations can be summarized as follows:

a) Limited accuracy of about $30 \mathrm{~cm}$ at best, which doesn't serve PPS applications needs.

b) Inherently, limited signal availability in urban environment and in underground tunnels. [9]

c) Problems pertaining to TTFF even with advanced technologies like A-GPS, DGPS, LTO, SBAS ... etc.

d) Satellite-Based systems are too centralized and lack the desired localized control.

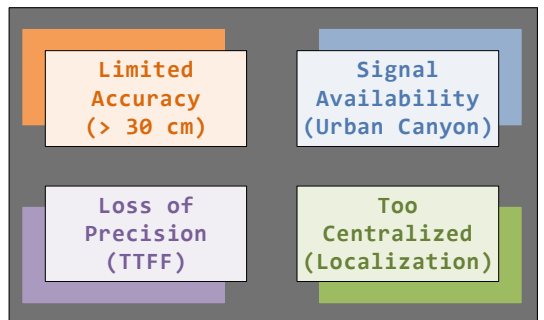

Limitations of GNSS Systems 
In urban environments, where public transit fleets tend to operate, satellite-based positioning systems like DGPS suffer from noise, the urban canyon syndrome and driving under bridges, in tunnels, through indoors parking facilities or indoor transit garages and maintenance facilities where satellite become inaccessible. There have been several past research efforts to improve current satellite-based positioning systems in harsh or difficult urban environments.

One approach attempts to extend the DGPS services into noisy environments by improving signal filtering[4]. Another studied the urban canyons syndrome and proposed a mechanism that utilizes two satellite signals instead of three. Those studies merely resolve one problem at the expense of another [8]. Solutions to the urban canyon problem come at the expense of accuracy ( $>>15$ meter). Satellite-Based solutions are not expected to reach the desired accuracy for PPS, certainly not in the near future and highly questionable if it ever will. For detailed description of the limitations of Satellite-Based Solutions, please refer to the Technical Approach Chapter.

\subsection{Medium to Short-Range Solutions}

Currently, Wi-Fi is getting the highest buzz as the most likely alternative positioning candidate. Wi-Fi provides highly desired combination of acceptable accuracy, high levels of geographical availability, short fix times, and excellent coverage in metropolitan areas. Most of the Wi-Fi positioning technologies can be ported to 5.9 GHZ DSRC which makes 5.9 GHZ DSRC another valuable solution on the long term. Positioning accuracy developed by current Wi-Fi industry is not quite up-to the desired level of fraction-of-a-centimeter accuracy. However, Wi-Fi approach relies on the availability of too many hot-spots. The logic is that the higher the number of hotspots, the better the accuracy.

Cellular triangulation technologies are now surfacing as alternative mechanisms. Carriers have invested heavily to comply with E911 requirements and are now looking for ways to monetize their investment. Other technologies, such as Broadcast TV-based methods have the potential for alternative use due to its high level of penetration through walls and buildings which makes it very attractive for situations under tunnels and bridges.

Another solution is the Cell-of-Origin ( $\mathrm{CoO})$ which presents a good fall back option despite its low accuracy; it can be used as a standalone technology for sanity validation. This alternative technology can be used as defense mechanism against malicious attacks, but cannot be the main source for positioning information.

Skyhook Wireless has presented a hybrid XPS solution that is based on GPS, Wi-Fi, and CoO. The XPS has been licenses to a wide range of chipset vendors and is being used for handheld applications. For the complete discussion of Medium to Short-Range Solutions, please refer to the Technical Approach Chapter.

\subsection{Embedded Road Markers}

It is important at this point of the report to distinguish between absolute and relative object location.

1) Absolute object location: defines object location in terms of longitude, latitude, and possibly, altitude with an error of less than $1 \mathrm{~cm}$. 
2) Relative object location: defines object location in terms of distance relative to other known local objects with an error of less than $1 \mathrm{~cm}$.

Most of the methods described thus far are focused on calculating the absolute object location. Embedded road markers approach is focused on relative object location. Fortunately, PPS works with either type of location methods. None of the previous methods achieved any accuracy close enough to the desired PPS operational accuracy of less than $1 \mathrm{~cm}$ of error.

On the other hand, embedded Road Markers are group of solutions that propose to embed markers on the road for the purpose of reading it later. Moving vehicle can then scan and position itself relevant to the road markers. There is no need to calculate the exact earth position, rather the relative object location from a known road mark (like curb, lane centerline, or bus-stop). The most known solution in this area is the California PATH Magnetic Guidance System (MGS) which is covered in the Technical Approach Chapter.

Other solutions such as passive RFID, active RFID, NFC (Near Field Communication), and UWB (Ultra Wide Band) are competing in the market of accurate location and positioning. It is unclear at this point in time which solutions will prevail. Active RFID and NFC seem to be most promising, with NFC being the most likely technology to be used in handsets. RFID positioning technologies are currently mainly developed for use in dedicated vertical applications such as logistics by companies such as Parco.

One advantage RFID and UWB hold over any other approach is the ability to embed road markers, yet maintain global positioning. Embedded RFID can include global positions as well as informing the moving vehicle about its relative position. Even more, RFID may include electronically embedded signs. For instance, an RFID may carry readable information about the road rules such as vehicle height limitations, approaching light signal, or left-turn rules. The RFID and UWB open the door for a deluge of applications.

Active RFID tags suffer from a limited range (hundreds of meters) and expensive chipsets, but can be tracked by Wi-Fi access points. Passive RFID systems provide cost-effective tags for short-range location solutions, but RFID readers and management software remain prohibitively expensive. None of the RFID technologies seem to be suited for wide-area location service.

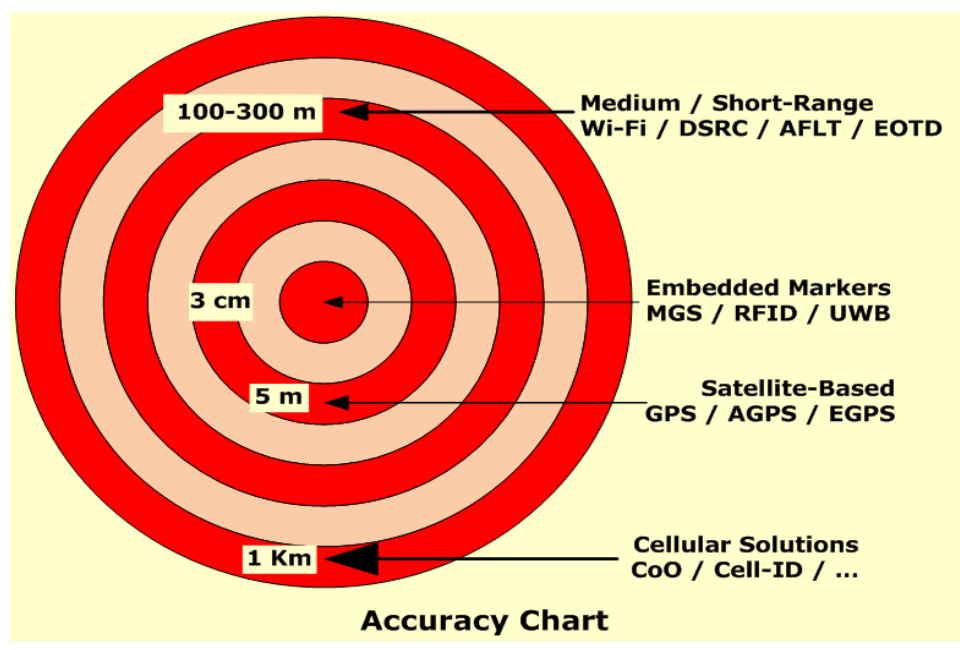




\section{Common Post-Processing Technologies}

Common add-on technology is a category of technologies that all proposed solutions may benefit from. A good example would be Dead-Reckoning, the use of motion sensors and context-aware positioning.

\subsection{Dead-Reckoning}

Traditionally, dead-reckoning sensors are used in car navigation systems to maintain positioning while driving through tunnels. The technology is based on the use of speed, acceleration, and direction sensors to calculate the position starting from a known reference point. This technology works well at higher speeds and for short periods. Current efforts are even extending efforts to adapt dead-reckoning technology in handsets and other mobile consumer devices. [6]Error!

\section{Reference source not found.}

Sensor Platforms is one of the companies developing dead-reckoning location-awareness sensors, such as accelerometers and micro-magnetometers. In 2008, Sensor Platforms offered solutions that can be integrated in handsets and personal navigation devices for improved indoor positioning. Dead-Reckoning needs a known start reference position; therefore, it is not a standalone positioning technology. Dead-Reckoning will play a role as a fallback option for deepindoor coverage when location signals are unavailable. Dead-Reckoning inherently accumulates errors over time. It is only usable for short periods of time and over short distances.

Orientation and direction sensors will be a key enabler for slow navigation as the lower speed (even zero speed) makes guidance difficult. Magnetometers permit knowledge of the vehicle's position and orientation under all circumstances. Currently, car manufacturers are loading vehicles with more motion sensors than ever. The motion sensor evolution is fueled by small hand-held devices. The iPhone contains a sensor to detect the portrait/landscape orientation of the device. Several Nokia phones contain motion sensors that can be used to collect fitness statistics while a user is jogging. The Nintendo Wii gaming platform allows game playing to be based on movements of the remote control. As motion sensor technology becomes a standard feature, it can be used at little extra cost for PPS purposes

\subsection{Context Awareness and Map Matching}

Another way to improve the observed accuracy is to apply context awareness. In that scenario, vehicles need to maintain continuous awareness of its surroundings using road maps. Then, filter out invalid positions such as positions outside a bridge or inside a building. Context awareness approach should be treated carefully since the filtration logic must accommodate unusual situations. Therefore, context awareness must include general knowledge about the status of the vehicle in general. Knowing that the air-bags have been deployed may suggest the vehicle has hit an obstacle, and the filtration logic must accommodate that by maintaining full context from all vehicle sensors, or refrain from changing observed location.

Context awareness and map matching efforts are still in their infancy. Currently, most efforts in this regard are focused on in-doors applications where the knowledge of a building or a garage map is clear. Also the signal availability can be studied to improve the process of weighing the confidence in the observed location. 
International Journal of Information Technology Convergence and Services (IJITCS) Vol.5, No.5, October 2015

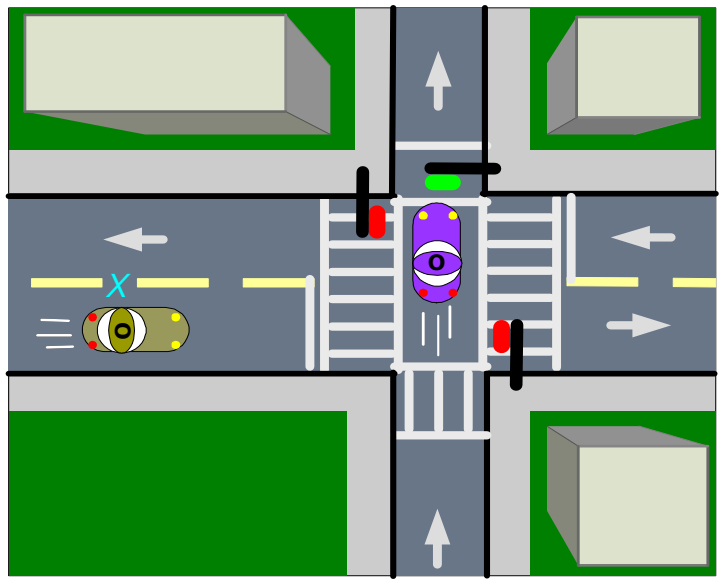

\subsection{Technical Comparison}

Table1: Technical Comparison of Alternative PPS Approaches

\begin{tabular}{|c|c|c|c|c|c|c|c|c|c|c|c|c|c|c|}
\hline & \multicolumn{3}{|c|}{$\begin{array}{l}\text { Satellite } \\
\text { Based }\end{array}$} & \multicolumn{8}{|c|}{$\begin{array}{l}\text { Medium and } \\
\text { Short-Range Wireless }\end{array}$} & \multicolumn{3}{|c|}{$\begin{array}{c}\text { Embedded } \\
\text { Road } \\
\text { Markers }\end{array}$} \\
\hline & है & $\frac{0}{6}$ & 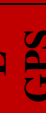 & 8 & ย & \& & 통 & 皇 & $\frac{1}{3}$ & है & $\frac{\overline{0}}{0}$ & $\frac{2}{2}$ & 空 & $\sum^{\infty}$ \\
\hline Localization & -3 & -3 & -3 & +1 & +1 & +1 & +1 & +1 & +1 & -2 & N/A & +3 & +3 & +3 \\
\hline Redundancy & -3 & -2 & -1 & -3 & -1 & -1 & -1 & -1 & +1 & +1 & N/A & +3 & +3 & +3 \\
\hline Accuracy & +2 & +2 & +2 & -3 & -3 & -3 & -3 & -3 & -3 & -3 & -3 & +3 & +3 & +3 \\
\hline suburban & +1 & +1 & +1 & -3 & -3 & -3 & -3 & -3 & -3 & -3 & -3 & +3 & +3 & +3 \\
\hline urban & $\mathbf{0}$ & $\mathbf{0}$ & $\mathbf{0}$ & -2 & -2 & -2 & -2 & -2 & -2 & -2 & -2 & +3 & +3 & +3 \\
\hline urban canyon & -1 & -1 & -1 & -1 & -1 & -1 & -1 & -1 & -1 & -1 & -1 & +3 & +3 & +3 \\
\hline awareness & -3 & -3 & -3 & -3 & -3 & -3 & -3 & -3 & -3 & -3 & -3 & -3 & -3 & -3 \\
\hline Reliability & +3 & +3 & +3 & +2 & +2 & +2 & +2 & +2 & +1 & +2 & +2 & +2 & +2 & +2 \\
\hline $\begin{array}{l}\text { Fault } \\
\text { Tolerance }\end{array}$ & -1 & -1 & -1 & -1 & -1 & -1 & -1 & -1 & -1 & $\mathbf{0}$ & N/A & +3 & +2 & +2 \\
\hline Cost & +3 & +3 & +3 & +1 & +1 & +1 & +1 & +1 & +2 & +2 & $\mathbf{0}$ & -2 & -2 & -2 \\
\hline installation & +1 & +1 & +1 & +1 & +1 & +1 & +1 & +1 & +1 & -1 & $\mathbf{0}$ & -2 & -2 & -2 \\
\hline operation & +3 & +3 & +3 & +2 & +2 & +2 & +2 & +2 & +2 & +1 & 0 & -1 & -1 & -1 \\
\hline maintenance & +3 & +3 & +3 & +1 & +1 & +1 & +1 & +1 & +2 & +1 & $\mathbf{0}$ & -2 & -2 & -2 \\
\hline $\begin{array}{l}\text { Future } \\
\text { Prospect }\end{array}$ & +3 & +3 & +3 & +1 & +1 & +1 & +1 & +1 & +2 & +1 & $\mathbf{0}$ & -2 & -2 & -2 \\
\hline short-term $<10$ & $\mathbf{0}$ & $\mathbf{0}$ & $\mathbf{0}$ & -2 & -2 & -2 & -2 & -2 & -1 & -2 & $\mathbf{0}$ & +3 & +3 & +3 \\
\hline long-term $>10$ & +2 & +2 & +2 & $\mathbf{0}$ & $\mathbf{0}$ & $\mathbf{0}$ & $\mathbf{0}$ & $\mathbf{0}$ & +2 & -1 & $\mathbf{0}$ & +1 & +1 & +1 \\
\hline Legends & $\begin{array}{r}\text { Extr } \\
\text { De } \\
\end{array}$ & $\begin{array}{l}\text { mely } \\
\text { ired }\end{array}$ & & & $\overline{\mathrm{De}}$ & & Mo & rate & Unc & ired & $\begin{array}{r}\text { Hig } \\
\text { Unde } \\
- \\
\end{array}$ & $\begin{array}{l}\text { ly } \\
\text { ired }\end{array}$ & & $\begin{array}{l}\overline{\text { nely }} \\
\text { ired } \\
\end{array}$ \\
\hline
\end{tabular}


Following the brief discussion of the different PPS technologies, it is important to compare the potential of each technology. In the following comparison, we categorized each technology on a 7 levels ladder scale with the top being extremely desired and the bottom being extremely undesired. Embedded Road Markers seem to be the best answer in the short term. It is certainly the least complex technology and can provide the desired accuracy.

\section{From Table 1 ,4.3.3 Technical Comparison}

Table 1, it is clear that embedded road markers are the clear choice on the short term. The major issues with road markers are concerns relevant to its cost. Long-term projection is much harder. Assuming that medium and short-range wireless solutions continue to improve in accuracy, we might reach the tipping point when they present accurate and economical solution.

Therefore, it is important to continue to follow-up on embedded road markers while keeping a wide eye on medium and short-range wireless solutions for any breakthrough that narrows the gabs between the two approaches and offers much sensible economics.

\section{MARKet Directions}

The main barriers for the uptake of precision positioning are accuracy, reliability, and cost. It is important to realize that all technologies presented here are evolving simultaneously towards higher accuracy. Each technology has valid potential in certain environment and serious drawbacks in other environments. Therefore, those technologies are likely to augment each other and operate together.

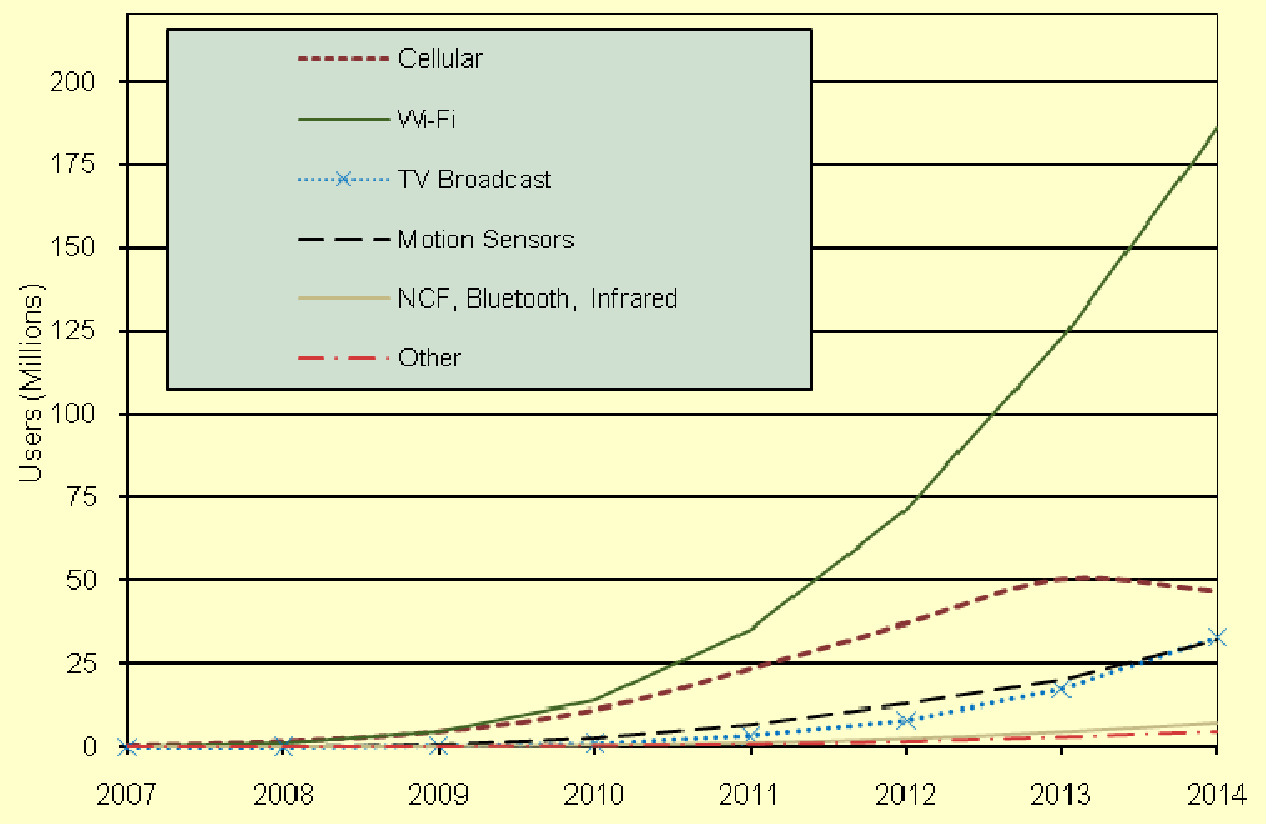

Chart 1: Alternative Positioning Technologies (APT) by Users (Source World Market) 
Other major considerations are the availability and existence of infrastructure. For instance, TVBroadcast and Cellular technologies are widely available throughout North America, which makes it very interesting to follow their evolution. Wi-Fi is quickly catching up with a growing number of hot-spots, yet the problem remains in building the right business model since Wi-Fi hot-spots are not typically controlled by a liable entities. Embedding road markers, on the other hand, seems to be the most accurate and quick solution, but the solution has economic drawbacks since the infrastructure is not in place.

To understand PPS market drives we have to investigate handset positioning technologies. A new breed of handset applications is called Location-Based Systems (LBS) and is currently driving the market of positioning systems. In order to predict the future of PPS we have to look at LBS evolution, and see how current positioning technologies are evolving from serving LBS towards PPS. The following charts illustrate the market growth of the current medium to short-range technologies. We focus on handset evolution and try to draw the parallel to the PPS projected market.

Chart 1 ,shows the evolution of the global number of users of each alternative positioning technology. Wi-Fi followed by Cellular solutions are the clear winners due to a combination of low costs, and wide availability.

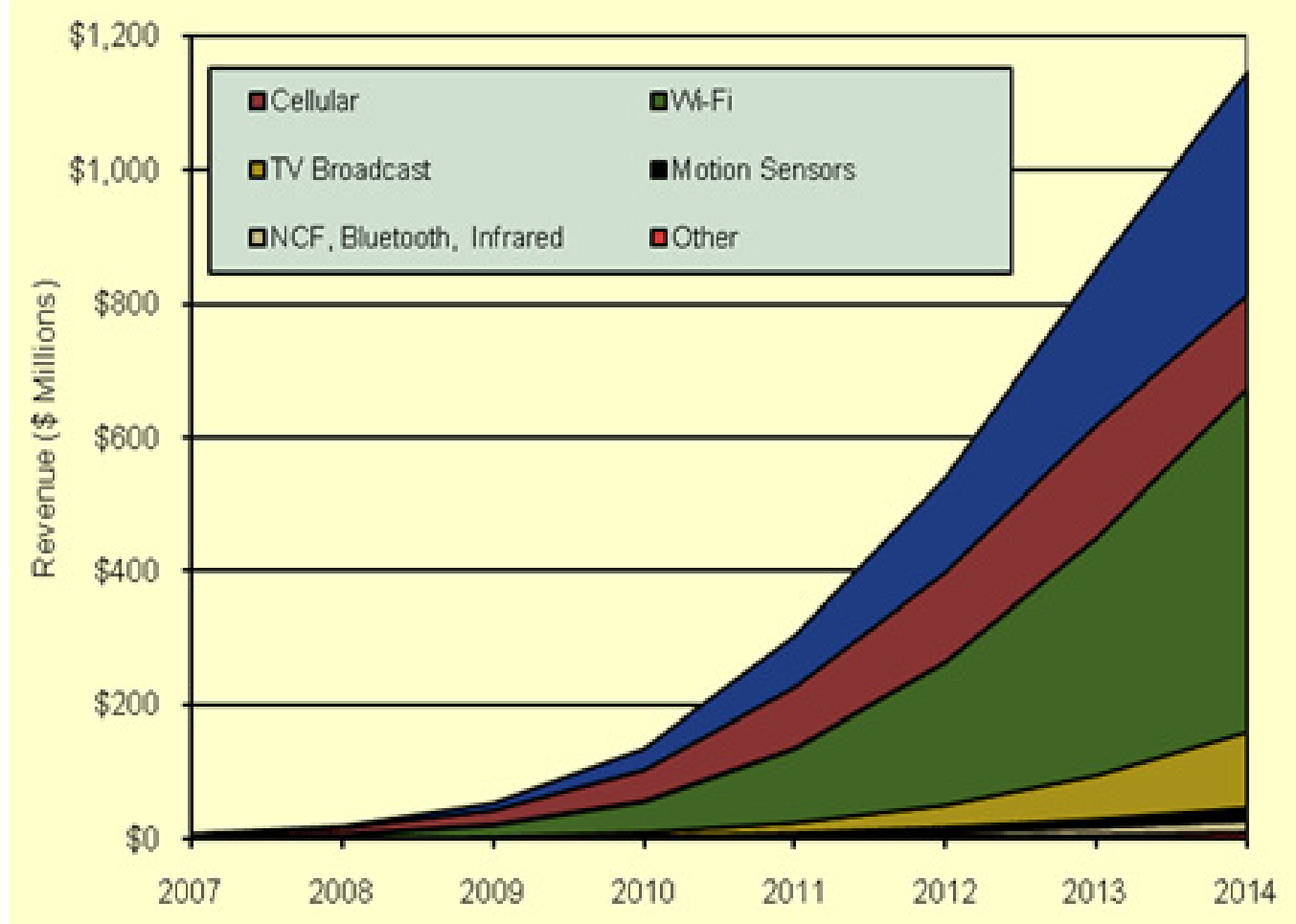

Chart 2 displays global alternative positioning licensing revenues. 
International Journal of Information Technology Convergence and Services (IJITCS) Vol.5, No.5, October 2015

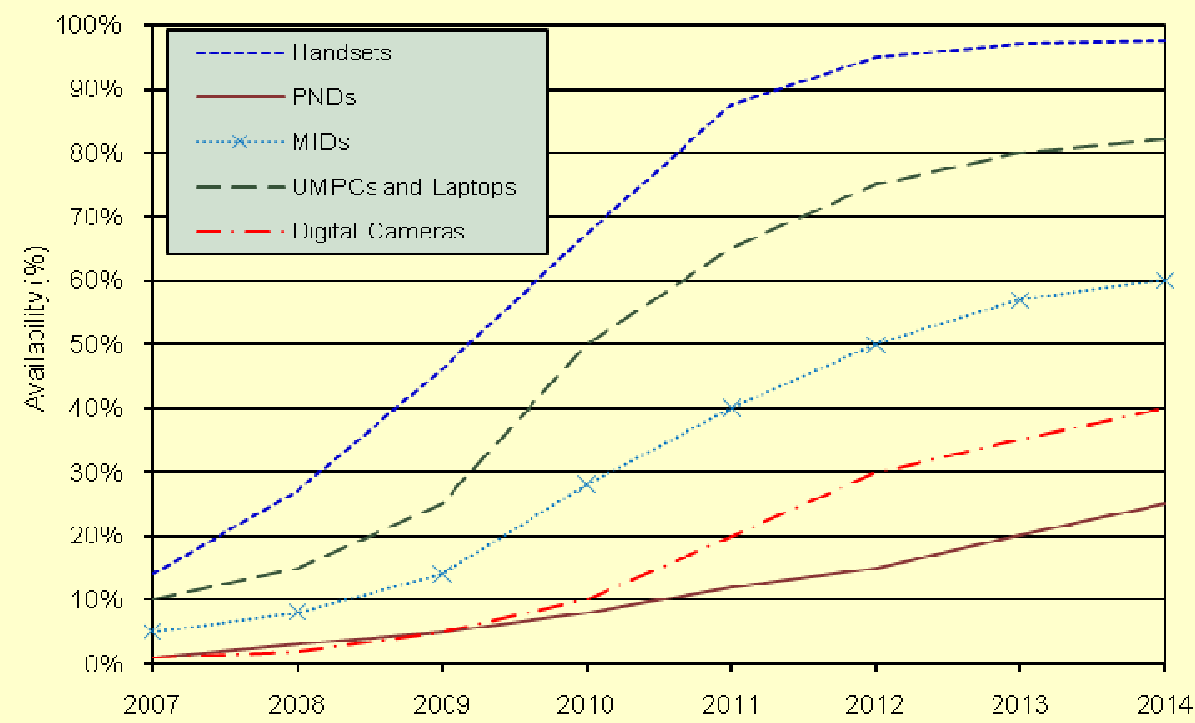

Chart 3: APTs by Device Availability from Factory (Source: RIM Market Study)

Chart 3,.shows alternative positioning technologies will first be implemented in handsets with other device categories showing a wide range of uptake and availability rates till 2014.

Alternative positioning technologies (APT) will initially be most popular on handsets applying modern Location Based Systems (LBS) like social networking applications, local search, and friend finders, but will eventually also be used for personal navigation and enterprise applications as shown in chart 4.

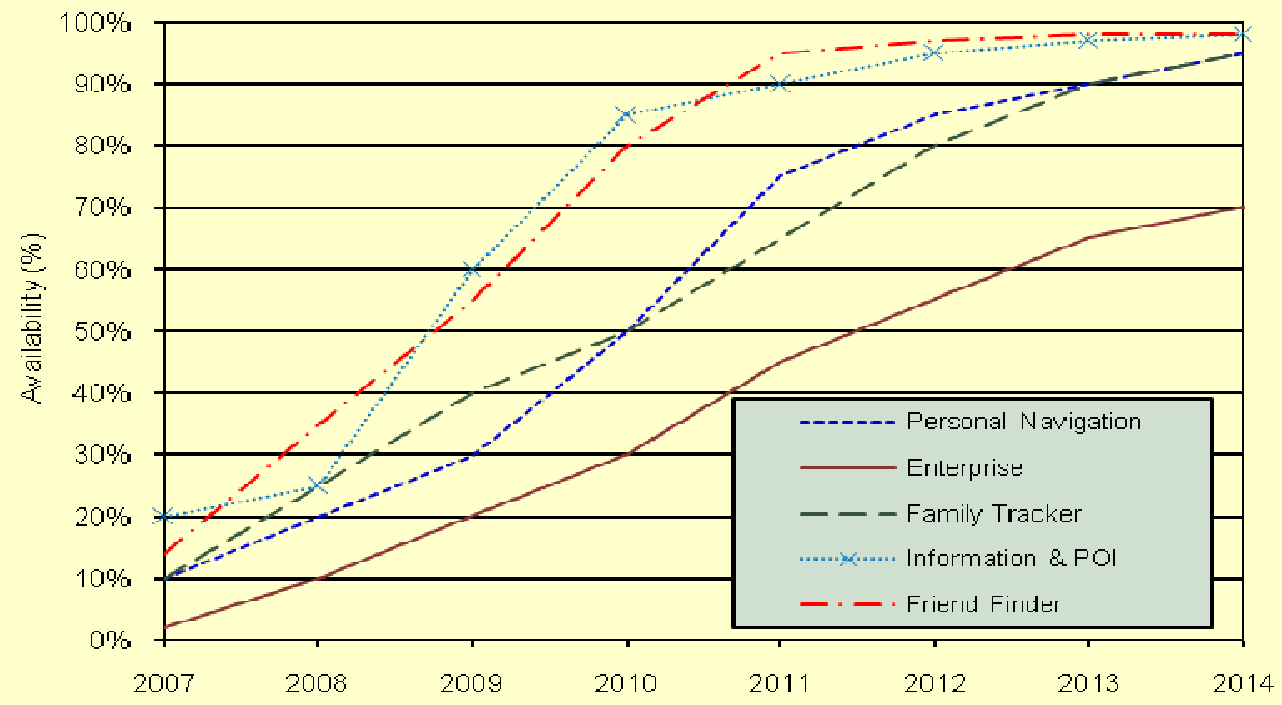

Chart 4: Apts By Lbs Application Type (Source: Ieee Communications Magazine) 


\section{Strategic Market Moves}

Accuracy, redundancy, and fault tolerance are some of the PPS key success factors along with cost effectiveness. Evidently, no single technology can provide perfect PPS uninterrupted in all situations. The positioning problem requires multiple technologies working together to provide accurate, reliable information at all times and in varying environmental conditions. It is not surprising that vendors are getting together to package PPS solutions based on their combined core competence. For instance, an agreement between Skyhook Wireless, GPS chipset vendors Broadcom, Qualcomm, SiRF, CSR, and Mobile OS vendor Symbian was reached late 2007 to investigate a complex blend of solutions. Those types of market moves represent very important steps towards making interoperable positioning a reality in the near future.

LBS have strong relevance to PPS and can be seen as the predecessor of PPS. New technologies, such as Wi-Fi and even $\mathrm{CoO}$, allow LBS providers to offer services independently from carriers. What is at stake is nothing less than the keys for dominating the location industry. Many parallels can be drawn with PPS in this regard. It is important to highlight here the creation of location vendor market as a new business model which can be replicated in the PPS market.

While major vendors, such as Google and Nokia, are building their own Wi-Fi reference databases via self-learning, user-generated content mechanisms due to their size and market share, smaller third-party location providers will typically buy alternative positioning databases.

Alternative positioning technologies pose yet another challenge to carriers hoping to avoid being relegated to mere bandwidth providers as compared to being a dominant and controlling element in the location value chain as the provider of positioning infrastructure and services. A new type of player is emerging in the form of location aggregators, such as LOC-AID and WaveMarket, centralizing and making location data easily accessible to service developers. This provides an opportunity for carriers to wholesale their location platforms and capabilities, which was recently confirmed by both Verizon and Sprint deciding to open up their networks and make location data available to aggregators and other third-party vendors.

The expected availability of public, free reference databases of cell tower locations and Wi-Fi, collected via self-learning processes based on user-generated feedback, endangers the monetization opportunities of solutions offered by commercial companies. Skyhook and other carriers are still trying to recuperate investments in location infrastructure imposed by the E911 mandate in the United States by offering commercial LBS applications. Cellular technologies might turn out to be too expensive for carriers to maintain and could be abandoned if they fail to generate sufficient revenues.

The future is bright for Wi-Fi and Cellular since they can be built independently by service providers, such as Google and Nokia, based on self-learning principles. Free Cell-ID-based location is enticing for free ad-funded social networking sites that cannot afford incurring any additional costs. Alternative positioning may become an open playing field even before it has reached full maturity. At the hardware level, motion sensors and electronic compasses will become more important for enabling pedestrian navigation. Broadcast TV and short-range technologies, such as Bluetooth or NFC, have not become significant location technologies within the forecast timeframe of this study. [1] [2] 


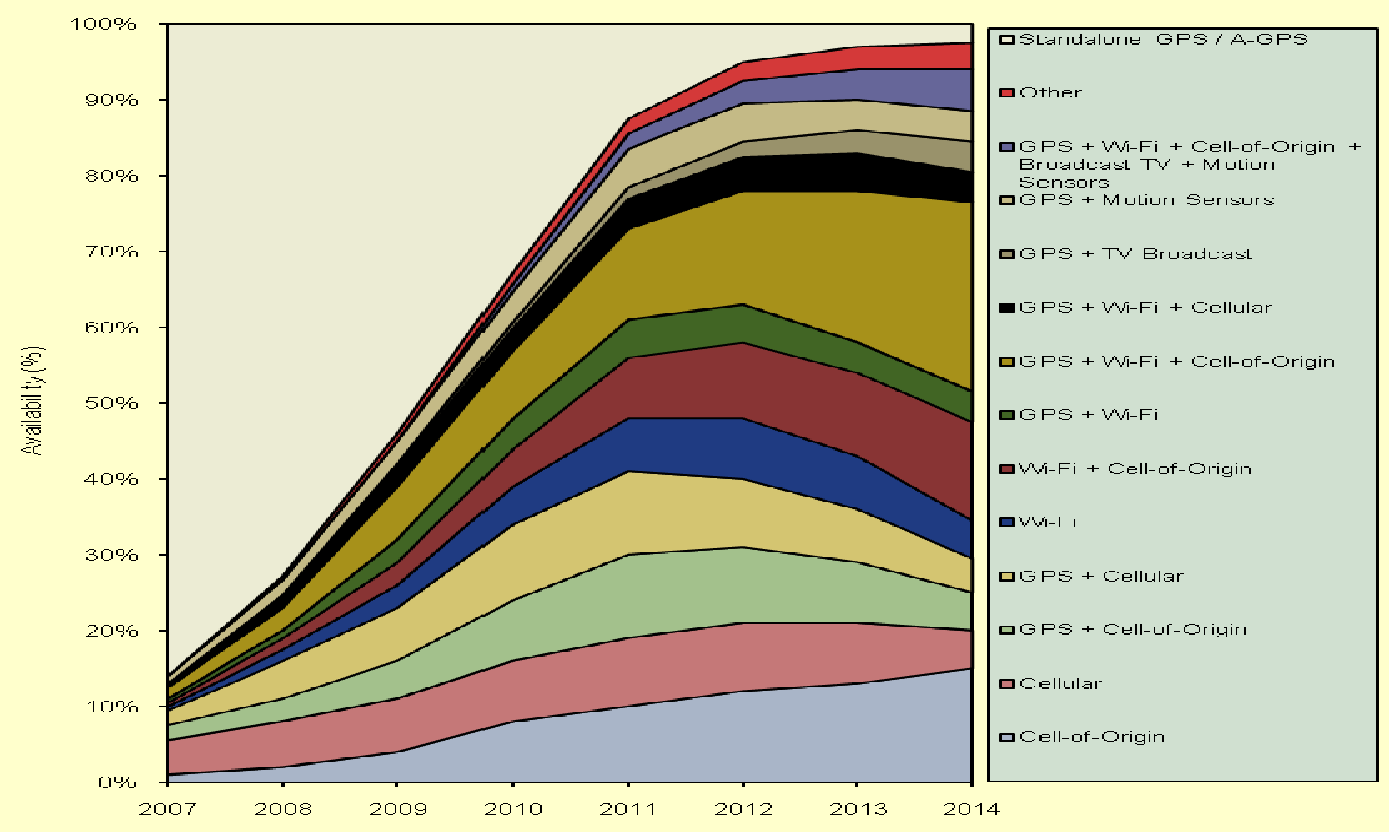

Chart 5: APTs Combinations Share Forecast (Source: World Market)

The availability of indoor location technology will prompt a range of innovations including pedestrian navigation and advanced LBA (Location-Based Advertising) in the years to come, but will also create new requirements, such as the availability of indoor digital maps.

Chart 5 ,shows which combinations of positioning technologies will be most successful with GPS $+\mathrm{Wi}-\mathrm{Fi}+\mathrm{Cell}-\mathrm{ID}$ and $\mathrm{Wi}-\mathrm{Fi}+\mathrm{Cell}-\mathrm{ID}$ as the winning combinations.

\section{Conclusion}

One can project that multiple technologies will eventually co-exist. Roaming vehicles would be able to obtain their position location from different sources, and then, consolidate and filter-out available information to come-up with the best estimate of its precise location. It is hard to completely dismiss one technology since it could offer more accurate positioning in particular scenario or geographical location. [11]

In the short run, we expect pilot projects to roll-out using embedded road markers. Those pilot projects would be focused on understanding the technology and the operations pertaining to running PPS solutions. In particular, we can see a great interest in applying embedded road marker solutions in transit ways of big cities as an attempt to harness the benefit of PPS. Pilot projects may serve particular areas, but there are no expectations of full deployment throughout cities. [6][10]

However, the long-term prospect is quite different. As the accuracy and reliability of medium and short-range wireless solutions improve, vehicular PPS market will be flooded by wireless solutions. The exact scenario of whether wireless solutions will take over embedded road markers or the other way around is hard to predict. Though, it is possible to envision the evolution of wireless positioning technologies by reading the charts provided in the previous section. 
It is expected that the current evolution in LBS will continue to influence the development of PPS. The crystallization of Location Providers as a viable business model is pivotal to easing the changes shaping up PPS. Eventually, a combination of Wi-Fi and Embedded Road Markers are expected to coexist and cooperate in providing PPS solutions.

\subsection{The Urban Transit Evolution}

Perhaps the most obvious beneficiary of PPS is the urban transit industry. Embedded road markers would fit the urban transit needs perfectly. Early road markers application will use dedicated transit-ways as the play ground and test-bed for optimization. The main driving force would be efficiency and operational cost effectiveness. For example, precision docking could potentially negate the need to deploy wheelchair ramps and make passenger queuing more efficient. Moreover, the ability to more precisely control the movement of the bus reduces the width of the lane required for travel from 3.65 meters - the current standard - to 3.00 meters. Other potential applications include automating bus passage through narrow tollbooths and vehicle routing in bus maintenance yards. The technology allows for a bus to safely follow closely behind another. Extra vehicles, much like extra cars on light rail trains, could thus be added during peak commute times. Many cities throughout the world, including 20 in the United States, have deployed some form of Bus Rapid Transit (BRT), although only a few include dedicated bus-only lanes.

\subsection{Canadian Context}

Unique weather and environmental conditions in Canada demands that Canadian researchers tweak and adjust solutions to fit our needs. Satellite-based solutions suffer from lower coverage as we go further north. GPS solutions also experience significant accuracy degradation in mountainous regions. Infrared fails measurably in snowy conditions. Embedded road markers seem to be the most promising alternative in terms of offering the desired accuracy. However, MGS bears some drawbacks when viewed from Canadian context:

1) The maximum spacing between MGS markers is 1.0 to 1.2 meters which is significantly small. While the cost of markers are generally low (<US\$ 0.90), wide spread deployment and maintenance cost remains prohibitively high.

2) The effects of road salt, snow, and snow removing operations have been loosely investigated. A thorough and comprehensive investigation of MGS operation on Canadian transit-ways is vital to evaluating the feasibility of MGS in Canadian context.

The two drawbacks of the MGS fuel the need for more research on improving MGS and alternative embedded road markers like RFID. Both approaches require further investigation and study. A pilot project could be the launching pad for evaluating embedded road markers within the Canadian context.

Potential applications of PPS extend beyond transit systems. PPS can be used in precision docking for trucks leading to faster operations at industrial platforms. Same applies to operations in marine ports and extends even to airplane docking. The speed, accuracy, safety and unmanned operation are so valuable and cannot be overlooked. [7] 


\section{RESEARCH FoCUS AND FUTURE WORK}

Despite the great research and industrial interest in PPS, available technologies are currently in their infancy. Existing solutions have yet to overcome many obstacles to fulfill common market demands. Applied research in preparation of deployment, proof of concepts and operational evaluations are essential to improve, alter, and optimize modern technologies to realize new market potential.

We can see the following valuable projects as a necessary continuation to this study:

1) Initiating a proof of concept project for enhancing Wi-Fi and 5.4 GHz DSRC PPS. Wi-Fi in particular offers the most promising solution of them all. Most of the solutions implemented on cellular technologies throughout the last decade can be ported to Wi-Fi after applying thoughtful and clever tweaks. The $5.4 \mathrm{GHz}$ DSRC presents another solution that holds most of the characteristics of Wi-Fi since DSRC is built over the WiFi architecture. Other advantage to Wi-Fi and DSRC solutions are cost effectiveness, localization and accuracy. The downside would be the availability of Line-of-Sight in DSRC, but the widespread use of those technologies would simply counter this argument. Therefore, investing in Wi-Fi and DSRC exploration towards PPS type of solutions presents an exceptionally wise and worthy approach. The proof of concept would capitalize on previous work and would require special lab equipments.

2) Establishing a proof of concept project on RFID-PPS. Most of the arguments against Magnetic Guidance Systems (MGS) can be countered if RFID were to be used as an alternative. The development of RFID has shown that it can be manufactured in smaller and smaller sizes and some RFIDs can be as small as the tip of a pencil. The size of the RFID has a great value in making deployment easier and more cost effective compared to MGS. Contrary to MGS, RFID can provide more information than just positioning. As an example, RFID may use embedded messages to direct the vehicle on a sharp curve, a situation that MGS cannot resolve without adding more road markers. RFID can also support security by adding signature to its embedded messages. Passive RFID can be of particular interest as since they do not need battery changes. Preliminary investigation of alternative RFID PPS solutions has been favorable and investment in a proof of concept is timely and much needed at this stage. In this project, collaboration with industrial players would add a great value.

3) Initiating a proof of concept project for implementing UWB technology on PPS. UWB uses very low energy levels for short-range high-bandwidth communications. UWB was designed in part for sensor data collection, precision locating and tracking applications. UWB is used as a part of location systems and real time location systems. The precision capabilities combined with the very low power make it ideal for certain radio frequency sensitive environments such as urban outdoor environment. Another benefit of UWB is the short broadcast time which enables implementers of the technology to install orders of magnitude more transmitter tags in an environment relative to competitive technologies. UWB does not suffer from the Line-of-Sight problem; instead, it may use see-throughthe-wall precision radar imaging technology. UWB uses precision locating and tracking (using distance measurements between radios), and precision time-of-arrival-based localization approaches. UWB can be combined with RFID to provide solutions that combine radar with precision positioning. 
4) Establishing and leading consortium foe defining PPS interoperability mechanisms. In a typical deployment, multiple PPS technologies will operate independently to come up with multiple estimates of the accurate position. Post processing is exceptionally important to weigh the multiple estimates, compare, filter and validate against each other or by applying map matching to come up with most sensible position estimate. In order to enable post processing technologies, it is vital to (1) associate each position estimate with a confidence value that express the confidence in the suggested estimate given the location and the environment; (2) build comprehensive policy as to how to evaluate each position estimate and how to combine the most sensible position estimate. Post processing would be a major challenge in the absence of common open platform that defines how multiple PPS systems may co-exist. For instance, despite the long life of GPS technologies, there is no known approach to defining a confidence value for the observed location. This particular topic requires the establishment of forums and industrial consortium and can be lead by government initiative.

5) Establishing operational applied research field testing and evaluation of the current MGS pertaining to the Canadian environment such as icy condition, effect of salt and snow removing, evaluating precision in harsh weather conditions, etc. This project can be held in close coordination with municipalities. A pilot project in the GTA area can be valuable for evaluating the MGS application in Canadian environment as well as improving the GTA transit system.

Given the Transport Canada interest in IntelliDriveSM (VII) the next step would be to focus on projects number one and two in the bullets above. The applications of precision positioning goes beyond public transit services as it might seem. It is vital to realize the wider range of PPS applications in marine port operations, airport operations, trucking industry, border clearance and many other applications. PPS presents the key element in unleashing the next generation of ITS applications pertaining to IntelliDriveSM (VII) and modern transportation safety applications. PPS is a fundamental element in automation and modern electronic highways. Backing pioneering efforts on PPS will likely result in spin-off developments in the transportation safety application field that would eventually improve the way we manage and run transportation highways.

\section{REFERENCES}

[1] H. Ahmed, M. EL-Darieby, B. Abdulhai, Y. Morgan "Bluetooth- and Wi-Fi-Based Mesh Network Platform for Traffic Monitoring," in the proceedings of the Transportation Research Board Annual General Meeting, Paper \#08-1848, January 2008.

[2] M. Pan and Y. Tseng "The Orphan Problem in Zigbee-based Wireless Sensor Networks," in the proceedings of the 10th ACM Symposium on Modeling, Analysis, and Simulation of Wireless and Mobile Systems, pp: 95-98, Chania-Crete Island, Greece, October 2007.

[3] Gallup Organization "General public survey on the European Galileo Program," Analytical Report by the Gallup Organization request from the DG Energy and Transport, Directorate R Resources, Information \& Communication, Hungary, June 2007.

[4] H. Tan and J. Huang "DGPS/INS-based Vehicle Positioning with Novel DGPS Noise Processing," in the Proceedings of the IEEE American Control Conference, pp. 3966-3971, Minneapolis MN-USA, June 2006.

[5] World Market Report: “A Market Review of Positioning Technologies," An IEEE report available for members of IEEE/IEEE-SA. 
International Journal of Information Technology Convergence and Services (IJITCS) Vol.5, No.5, October 2015

[6] US Department of Transportation, National Highway traffic Safety Administration, "Vehicle Safety Communications Project" Final Report, April 2006.

[7] National Center for Statistics and Analysis, "Traffic Safety Facts 2003," Report DoT HS 809 767, Nat'l. Highway Traffic Safety Admin., U.S.-DoT, Washington, DC, 2004.

[8] C. Chan and H. Tan "Evaluation of Magnetic Markers as a Position Reference System for Ground Vehicle Guidance and Control," Research Report, Institute of Transportation Studies, California Partners for Advanced Transit and Highways (PATH), University of California, Berkeley, August 2003

[9] Y. Cui and S. Ge "Autonomous Vehicle Positioning with GPS in Urban Canyon Environments," in the Proceedings of the IEEE International Conference on Robotics \& Automation, pp. 1105-1110, Seoul, Korea, May 2001.

[10] Magnemotion Incorporated, "Vehicle Guidance and Switching via Magnetic Forces," Patent \# (WO/1999/033691) URL: http://www.wipo.int/pctdb/en/wo.jsp?wo=1999033691.

[11] NHTSA, ITS Joint Program Office, "Report to Congress on the National Highway Traffic Safety Administration ITS Program, Program Progress during 1992-1996 and Strategic Plan for 1997-2002". U.S. Department of Transportation, Washington, DC, January, 1997. 